tious physicists prefer to attribute it to a random seismic effect rather than to new astrophysics.

At the special Moriond session, Maurice Goldhaber of Brookhaven and the IMB experiment declared, 'there are small discrepancies between absolute time observations, which can probably be resolved. These data will certainly be squeezed for all they are worth for a long time - perhaps until the next close supernova comes along. The near agreement with theory for this awesome phenomenon must be considered as a triumph for the input sciences - nuclear physics, particle physics and astrophysics, and increases confidence in the Big Bang calculations'.
Meanwhile the sparse data has sparked off a frenzy of activity, with many interpretations based on the the details of the neutrino observations. However astrophysicist Dave Schramm warns of the dangers of pressing these meagre statistics too hard.

\section{Coming in from the cold}

The minimal electrical resistance of superconducting materials provides an 'easy' way to high currents if the associated cryogenics problems can be mastered - conventional superconductors require ambient temperatures close to absolute zero. The discovery of new superconducting materials operating at much higher (but still cryogenic) temperatures brings the potential rewards much closer.

This article, specially written for the CERN Courier by a specialist in the field, gives an inside story on these latest developments. Meanwhile fresh 'warm' superconductor news continues to arrive. $A$ team at Berkeley reports that an as-yet unnamed substance shows a dramatic drop in electrical resistance at about $-39^{\circ} \mathrm{C}$, a 'temperature close to a winter's day in Fairbanks, Alaska'.

\title{
New high temperature superconductors
}

\author{
by David C. Larbalestier
}

Enormous interest has been aroused by the report of Georg Bednorz and Alex Müller of IBM's Zurich laboratories late last year of high temperature superconductivity in a mixed oxide of lanthanum, barium and copper. (Superconductivity, the abrupt fall of electrical resistance at low temperatures, was discovered by Kamerlingh Onnes in 1911, using mercury at liquid helium temperatures $4.2 \mathrm{~K}$.) The report of possible superconductivity at around $30 \mathrm{~K}$ was initially received somewhat sceptically. The phase responsible for the superconductivity was quickly identified by a Tokyo group as a layered 'perovskite' of approximate composition $\mathrm{La}_{1.8} \mathrm{Ba}_{0.2} \mathrm{CuO}_{4}$.

These layered perovskites are related to better-known perovskites such as barium titanate, a ferroelectric. The Tokyo group and a group at Houston presented results of their work at the Materials Research Meeting in Boston in early December. They were able to document the two independent properties characteristic of superconductivity: zero resistance and flux exclusion. The scientific community was convinced and the hunt was on. Three months later, many hundreds of scientists are working on the new oxides.

The search for new superconductors with higher transition temperatures $\left(T_{c}\right)$ and with possible new mechanisms has always been an exciting one. However the previous advance dates from 1973 when workers at Westinghouse and at Bell Labs advanced $T_{c}$ from about 21 to $23 \mathrm{~K}$ with niobiumgermanium films. A large effort was subsequently put into synthesizing niobium-silicon which appeared to be the logical next step 


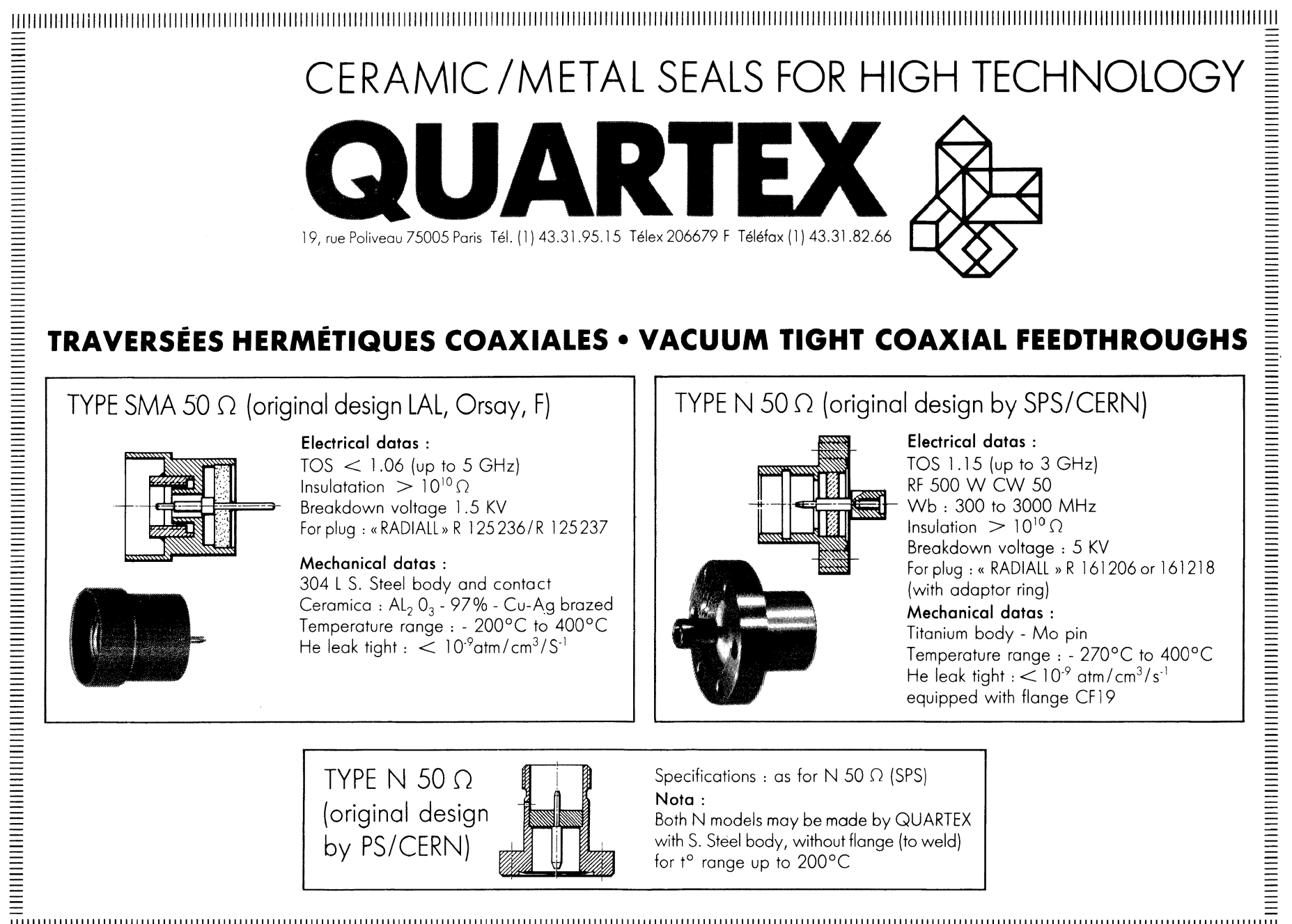

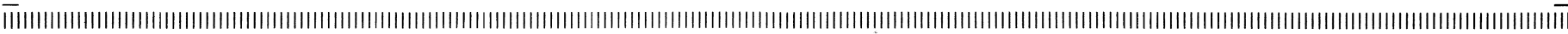

\section{NEUBERGER: THE PROMISE OF PRECISION}

Top quality

Top reliability

LS Display: fitted with analog inputs measure voltage and current (d.c., sinusoidal a.c. and true r.m.s.) and temperature via a $\mathrm{Pt}$ 100 probe and thermocouples (including compensation for cold welding).

Technical specifications

- Slim $144 \times 48$ mm format

- Horizontal or vertical mounting

- Scale markings in black or white

- Two galvanically separated inputs

- Class 1 precision

- Trigger or relay output

- Button or electronically controlled parameter settings

- Power supply: 12,24 , $11,220 \mathrm{~V} / 47 . .400 \mathrm{~Hz}$ $24 \mathrm{~V}$ d.c.

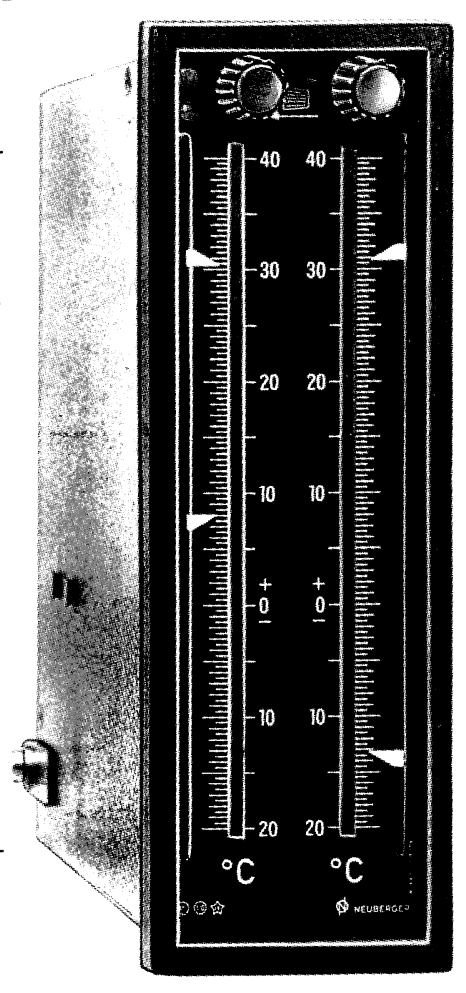

\section{MEGAMETRO SA}

Gentianes 24

$\mathrm{CH}-2300$ La Chaux-de-Fonds

Tel: (039) 235465

Telex 952263

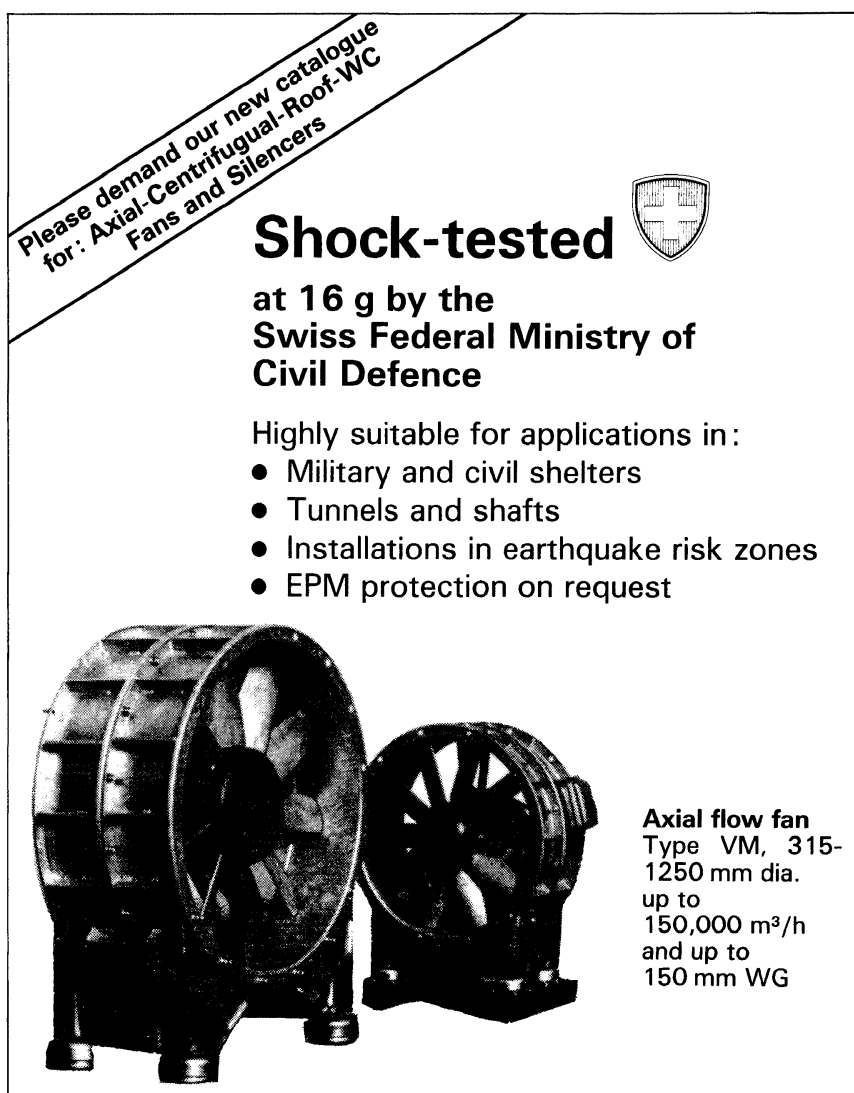

RRPIIIAG

Freigutstrasse $40, \mathrm{CH}-8002$ Zürich

Telephone 01/202 45 75, Telex 816093 
Crystal structure of one of the new 'warm' superconductivity candidates - the layered perovskite $\mathrm{La}_{1.8} \mathrm{Sr}_{0.2} \mathrm{CuO}_{4}$. The cell is tetragonal, the height of the cell being about 13.3 $A$ and the width of the cell about 3.8 A. The basal planes at top and bottom are believed to be those where superconductivity is favoured. These contain the copper and oxygen ions.

in the line from niobium-tin. Predictions of the $T_{c}$ of niobium-silicon ranged from around 25 to $35 \mathrm{~K}$. However it refused to form in the desired A15 crystal structure at the stoichiometric $(3: 1)$ composition and all efforts have so far proved fruitless. Reports of high temperature superconductivity in other more exotic compounds claimed $T_{c}$ values of greater than $100 \mathrm{~K}$ but none proved verifiable. After 15 years of static $T_{c}$, there was a feeling of scepticism about further advances. Possibly for this reason, Bednorz and Müller were modest in their initial claim. They observed zero resistance only at 12-13 K ; however they observed a distinct decline in resistance at around $30 \mathrm{~K}$ and they speculated this might be the signature of percolative superconductivity, where islands of superconductivity are weakly coupled together. Now we know that they were right.

Exciting as this report was, it was only the prelude to a more spectacular result. Following the Boston meeting, many groups took notice and many similar compounds (there are at least several hundred known layered perovskites) were fabricated. Very quickly a Bell group found that $\mathrm{La}_{1.8} \mathrm{Sr}_{0.2}$ $\mathrm{CuO}_{4-y}$ (the $\mathrm{y}$ is uncertain because there are an undetermined number of oxygen vacancies in the structure) had a $T_{c}$ of $36-40 \mathrm{~K}$. The Houston group found the original barium oxide to have an onset $T_{c}$ of up to $52 \mathrm{~K}$ under pressure and the race was really on. In early February, a joint team led by C. W. Chu (Houston) and M. K. Wu (Alabama) reported superconductivity at about $90 \mathrm{~K}$. Reports of this work appeared in Physical Review Letters on 3 March. The compound is similar to the La-Ba-Cu oxide, lanthanum being replaced by yt- trium. However, the proportion of barium is greater, $\mathrm{Y}_{1.2} \mathrm{Ba}_{0.8} \mathrm{CuO}_{4-\mathrm{y}}$ and the crystal structure is not yet determined.

The appearance of superconductivity at temperatures accessible to liquid nitrogen cooling (77K) is of course of tremendous interest. The low latent head of vaporization of helium $\left(2.9 \mathrm{~J} / \mathrm{cm}^{3}\right)$ and the low specific heat of materials at liquid helium temperatures make for instabilities. Liquid nitrogen has a much larger latent heat $\left(159 \mathrm{~J} / \mathrm{cm}^{3}\right)$ and is about ten times cheaper than helium. Superconducting magnets operating with liquid nitrogen should be very stable and it is natural to speculate whether a revolution in superconducting technology is imminent.

Given such dramatic discoveries and such a rapid advance, it is difficult to make a definitive view of the technological impact of these materials. However let me make some comments appropriate at the time of writing (5 March), with the reservation that developments in the following weeks and months may invalidate these remarks.

These materials appear to be BCS superconductors (that is, their properties appear to be explicable in terms of the traditional BardeenCooper-Schrieffer theory of superconductivity), albeit very strongly coupling ones. Strong coupling means the BCS picture is modified to make the basic Cooper pair coupling stronger. This is consistent with the atomic structure of the layered perovskite, in which the copper ions are surrounded by an octahedral oxygen array. The most favoured present explanation for the properties is that the substitution of a divalent cation such as $\mathrm{Sr}^{2+}$ for a trivalent $\mathrm{La}^{3+}$ ion produces partial transformation of

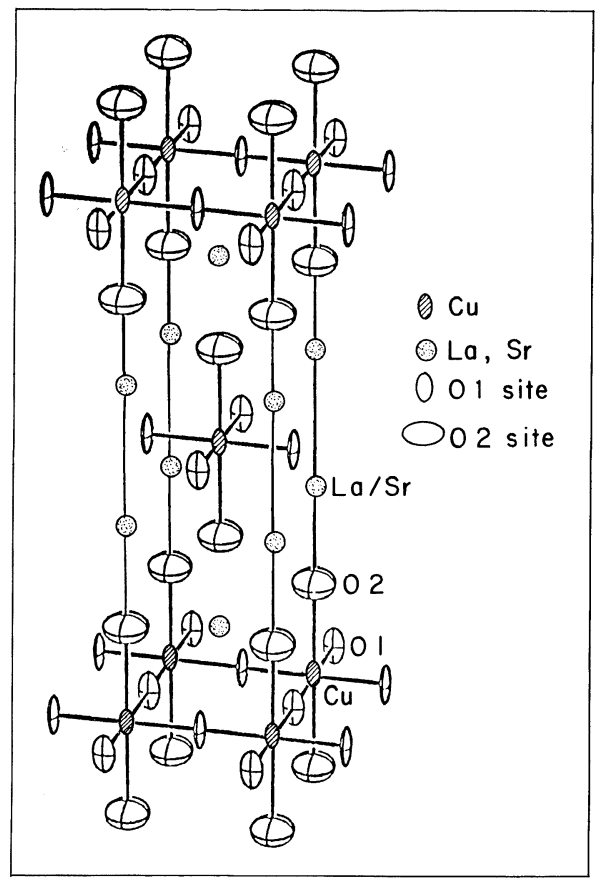

$\mathrm{Cu}^{2+}$ to $\mathrm{Cu}^{3+}$. The extent of this depends on the oxygen vacancy concentration; if no vacancies exist, each substitution of a $\mathrm{Sr}^{2+}$ ion converts a $\mathrm{Cu}^{2+}$ ion to a $\mathrm{Cu}^{3+}$ ion. The mixed valence state produces metallic conductivity; the unsubstituted $\mathrm{La}_{2} \mathrm{CuO}_{4}$ is a semiconductor. In this model, it is believed that a strong electron-phonon interaction exists, these conditions being favourable to high temperature superconductivity.

Given that they are BCS-like superconductors, we should expect high critical fields to go with the high critical temperatures. Many workers have now measured the critical field slope at $T_{c}$ to be of the order of $2 \mathrm{~T} / \mathrm{K}$ and rather sensitive to measurement conditions. According to standard theory, this indicates that the critical field at which superconductivity is destroyed could be around 50 to $150 T$ at $0 \mathrm{~K}$. No such DC fields presently exist to check the predictions. However measurements by a group at MIT and Bell do suggest at least $45 \mathrm{~T}$ at $4.2 \mathrm{~K}$. Unfortunately the transition is of order 20T wide.

A very broad transition is common to all the measurements presently known to me, including our own attempts to pass transport currents through the $\mathrm{Ba}$ and $\mathrm{Sr}$ oxides. Attempts to pass current densities of much more than about $10 \mathrm{~A} / \mathrm{cm}^{2}$ produce very extended transitions. The most favoured 


\section{Heraeus}

is your adress for

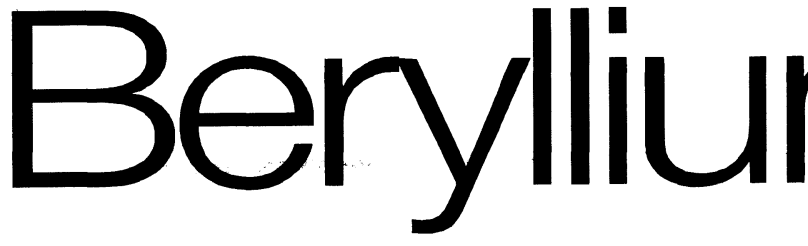

All companies have their specialities.

We are no exception with our long tradition in manufacturing special metals.

Heraeus has a vast experience in machining and fabrication of special metals such as Beryllium. Our purpose designed workshops are fully equiped to enable us to offer a comprehensive range of turned, milled, spark eroded and brazed products.

We look forward to receiving your equiries.

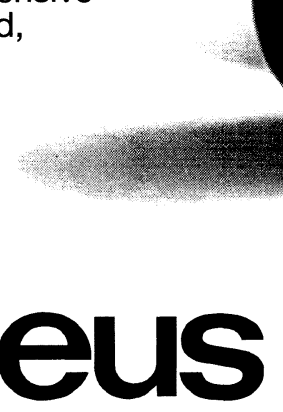

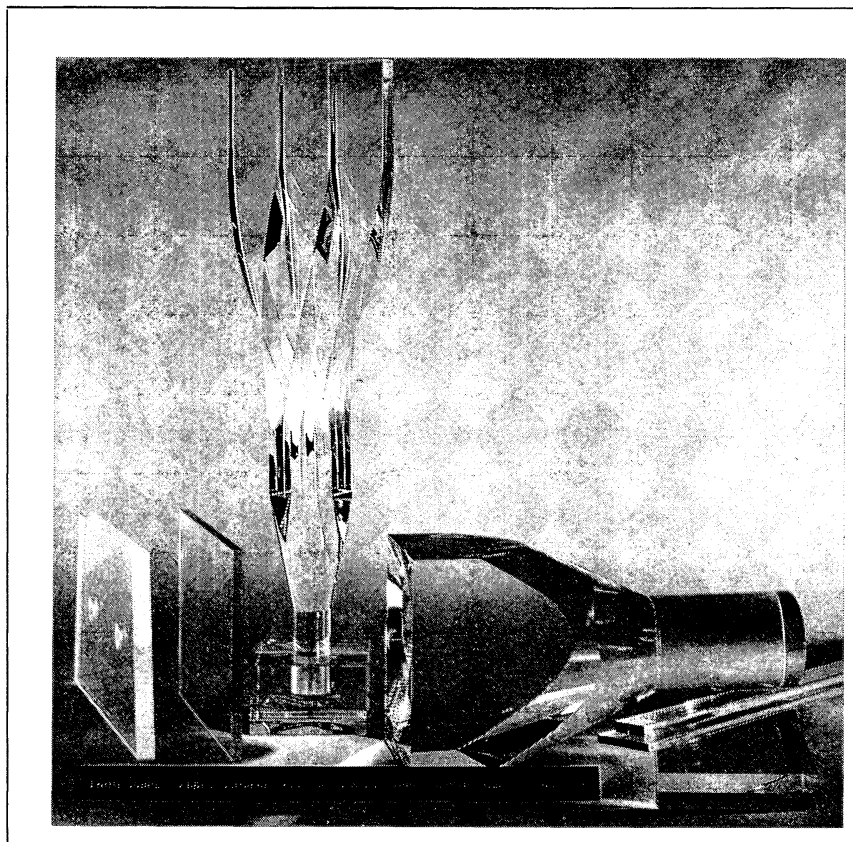

\section{POLIVAR S.p.A.}

Via Trieste 10/12 P.O. Box 111

00040-POMEZIA (Roma)

Tel. 06/912 1061 Telex 611227 PLVI

\section{Product line}

Cast acrylic sheets, bars, blocks, scintillators, light guides

\section{Dew point}

\section{transmitter}

\section{Measurements in}

\section{process technology}

air ducts meteorology

low cost

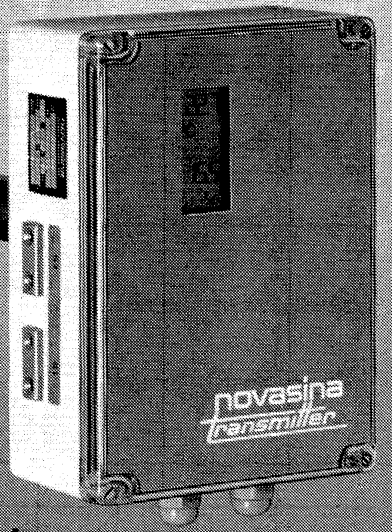

exchangeable measuring cells

automatic calibration

NOVASINAAAG

Talstrasse $35-37$

Fax 055/476262 CH-8808 Pfáffikon/SZ

Tel: $055 / 4765.65-T$ TX. 876:245 sina ch A company of WMH Walter Meier Holding AG 
present explanation for this behaviour is that superconductivity exists in many discrete regions within the sample but only weak coupling exists between these regions. This weak coupling is strongly diminished by an external field. Electromagnetic and structural characterizations of these compounds are proceeding furiously and we may soon expect to know whether this percolative nature of the superconductivity is inherent in the material or results from the way present samples are being prepared.
The long timescale needed to produce acceptable conductors of niobium-tin (10-12 years) following its discovery in 1962 inevitably comes to mind in assessing the potential timescales for developing high field magnets with the new materials. These new oxides, like niobium-tin and the other A 15 compounds are inherently brittle and this is bound to affect their application in magnets, just as large scale construction of niobiumtin magnets has lagged behind their ductile niobium-titanium coun- terparts. But superconductivity above $77 \mathrm{~K}$ provides a powerful stimulant to solve the technological problems of dealing with brittle materials. The next few months could give us many more surprises in an area where none were suspected only a few months ago. The discovery of Bednorz and Müller has given a profound new stimulus to superconductivity and the last discovery has surely not yet been made.

\section{The $A$ to $Z$ of accelerators}

With great skill, the organizers of the 1987 Particle Accelerator Conference arranged a vast programme to run through nearly 700 papers from Session A to Session $Z$. They took in en route the latest on construction and plans for the high energy machines for particle

US Superconducting Super Collider Central Design Group leader Maury Tigner - aiming at the highest possible energies.

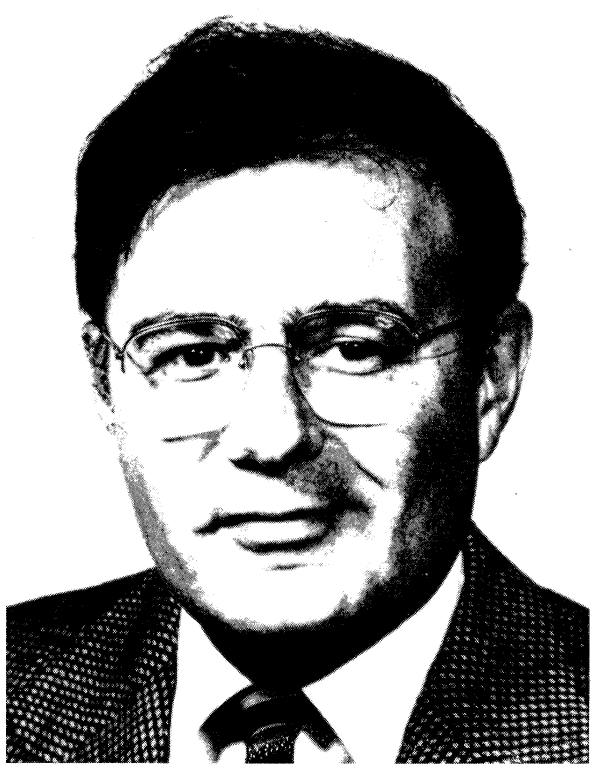

physics but this information was surpassed in volume by reports from the many other areas where accelerators now play a key role.

Synchrotron light sources are now illuminating the world in almost thirty Laboratories. Free electron lasers are confirming their promise for the creation of undreamed of fluxes of electromagnetic radiation. 'Star wars' machines are now openly discussed. Reports on other actual or potential applications in medicine, food processing, fusion, etc., underlined how the seemingly esoteric technology developed for the study of particles has continued to ripple into everyday life. (Already many people sit in front of a particle accelerator for several hours every evening!)

The Conference was held in Washington from 16-19 March and was the 12th in the North American series which began in

Giorgio Brianti - pushing for a hadron collider in the tunnel now being completed at CERN for the LEP electron-positron collider.
1965. The attendance of over 1100 was another reflection of the wealth of activity in the field.

The debate on the big machines

Since the hot topic appeared in Session Z, not many people left the Conference early. In the wake

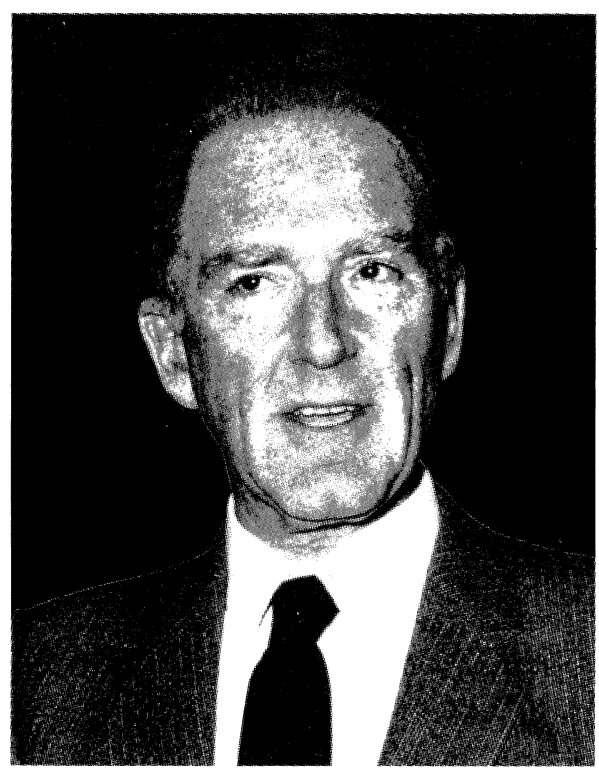

